\title{
On Flat Pseudo-Euclidean Nilpotent Lie Algebras
}

\author{
Mohamed Boucetta ${ }^{\mathrm{a}}$, Hicham Lebzioui ${ }^{\mathrm{b}, *}$ \\ ${ }^{a}$ Université Cadi-Ayyad, Faculté des sciences et techniques, B.P. 549, Marrakech, Maroc. \\ m.boucetta@uca.ac.ma \\ ${ }^{b}$ Université Moulay Smaïl, École Supérieur de Technologie Khénifra, B.P : 170, Khénifra, Maroc. \\ h.lebzioui@estk.umi.ac.ma
}

\begin{abstract}
A flat pseudo-Euclidean Lie algebra is a real Lie algebra with a non degenerate symmetric bilinear form and a left symmetric product whose the commutator is the Lie bracket and such that the left multiplications are skew-symmetric. We show that the center of a flat pseudo-Euclidean nilpotent Lie algebra of signature $(2, n-2)$ must be degenerate and all flat pseudo-Euclidean nilpotent Lie algebras of signature $(2, n-2)$ can be obtained by using the double extension process from flat Lorentzian nilpotent Lie algebras. We show also that the center of a flat pseudoEuclidean 2-step nilpotent Lie algebra is degenerate and all these Lie algebras are obtained by using a sequence of double extension from an abelian Lie algebra. In particular, we determine all flat pseudo-Euclidean 2-step nilpotent Lie algebras of signature $(2, n-2)$. The paper contains also some examples in low dimension.
\end{abstract}

Keywords: Nilpotent Lie algebras, Nilpotent Lie groups, Flat left-invariant metrics, double extension.

2010 Mathematics Subject Classification: 17B60; 17B30; 17B10; 53C50.

\section{Introduction}

A flat pseudo-Euclidean Lie algebra is a real Lie algebra with a non degenerate symmetric bilinear form and a left symmetric product whose the commutator is the Lie bracket and such that the left multiplications are skew-symmetric. In geometrical terms, a flat pseudo-Euclidean Lie algebra is the Lie algebra of a Lie group with a left-invariant pseudo-Riemannian metric with vanishing curvature. Let $(\mathfrak{g},\langle\rangle$,$) be a flat pseudo-Euclidean Lie algebra of dimension n$. If the metric $\langle$,$\rangle is definite positive (resp. of signature (1, n-1)$ ), then $(\mathfrak{g},\langle\rangle$,$) is called Euclidean$ (resp. Lorentzian). Flat pseudo-Euclidean Lie algebras have been studied mostly in the Euclidean and the Lorentzian cases. Let us enumerate some important results on flat pseudo-Euclidean Lie algebras:

1. In [8], Milnor showed that $(\mathfrak{g},\langle\rangle$,$) is a flat Euclidean Lie algebra if and only if \mathfrak{g}$ splits orthogonally as $\mathfrak{g}=\mathfrak{b} \oplus \mathfrak{u}$, where $\mathfrak{u}$ is an abelian ideal, $\mathfrak{b}$ is an abelian subalgebra, and $\operatorname{ad}_{b}$ is skew-symmetric for any $b \in \mathfrak{b}$. According to this theorem, a nilpotent (non-abelian) Lie algebra can not admit a flat Euclidean metric.

${ }^{*}$ Corresponding author

Preprint submitted to Elsevier

July 19, 2018 
2. In [2], Aubert and Medina showed that all flat Lorentzian nilpotent Lie algebras are obtained by the double extension process from Euclidean abelian Lie algebras.

3. Guédiri showed in [7] that a flat Lorentzian 2-step nilpotent Lie algebra is a trivial extension of the 3-dimensional Heisenberg Lie algebra $\mathcal{H}_{3}$.

4. In [3, 4], the authors showed that flat Lorentzian Lie algebras with degenerate center or flat nonunimodular Lorentzian Lie algebras can be obtained by the double extension process from flat Euclidean Lie algebras.

The study of flat pseudo-Euclidean Lie algebras of signature other than $(0, n)$ and $(1, n-1)$ is an open problem. In this paper, we tackle a part of this problem, namely, we study flat pseudoEuclidean nilpotent Lie algebras of signature $(2, n-2)$ and flat pseudo-Euclidean 2-step nilpotent Lie algebras of any signature. There are our main results:

1. In Theorem 3.1, we show that the center of a flat pseudo-Euclidean nilpotent Lie algebra of signature $(2, n-2)$ must be degenerate. From this theorem and Theorem 4.1 we deduce that all flat pseudo-Euclidean nilpotent Lie algebra of signature $(2, n-2)$ are obtained by the double extension process.

2. We give some general properties of flat pseudo-Euclidean 2-step nilpotent Lie algebras and we show that their center is degenerate. we show also that we can construct all this Lie algebras by applying a sequence of double extension starting from a pseudo-Euclidean abelian Lie algebra.

3. We give all 2-step nilpotent Lie algebras which can admit flat pseudo-Euclidean metrics of signature $(2, n-2)$ (Theorem 6.1 and Theorem 6.2). We will see that a class of 2step nilpotent Lie algebras which can admit a flat pseudo-Euclidean metrics of signature $(2, n-2)$ is very rich, contrary to the Euclidean and the Lorentzian cases. As example, we show that any 6-dimensional 2-step nilpotent Lie algebra which is not an extension trivial of a 5-dimensional Heisenberg Lie algebra, admits such metric.

The paper is organized as follows. In section 2, we give some generalities on flat pseudoEuclidean Lie algebras. In section 3 and section 4, we study flat pseudo-Euclidean metrics of signature $(2, n-2)$ on nilpotent Lie algebras . In section 5, we study flat pseudo-Euclidean 2-step nilpotent Lie algebra of any signature. In section 6, we give all flat pseudo-Euclidean 2-step nilpotent Lie algebras of signature $(2, n-2)$. We end the paper by giving some examples.

\section{Preliminaries}

In this section, we give some general results on nilpotent Lie algebras and on flat pseudoEuclidean nilpotent Lie algebras which will be crucial in the proofs of our main results.

Let us start with two useful lemmas. Recall that a pseudo-Euclidean vector space is a real finite dimensional vector space endowed with a non degenerate bilinear symmetric form.

Lemma 2.1. Let $(V,\langle\rangle$,$) be a pseudo-Euclidean vector space and A$ a skew-symmetric endomorphism satisfying $A^{2}=0$ and $\operatorname{dim} \operatorname{Im} A \leq 1$. Then $A=0$.

Proof. Suppose that $A \neq 0$. Then $\operatorname{Im} A$ is a totally isotropic vector space of dimension 1 . This implies that $\operatorname{ker} A$ is an hyperplan which contains $\operatorname{Im} A$. Let $e$ be a generator of $\operatorname{Im} A$ and choose an isotropic vector $\bar{e} \notin \operatorname{ker} A$ such that $\langle e, \bar{e}\rangle=1$. We have $V=\operatorname{ker} A \oplus \mathbb{R} \bar{e}$ and $A(\bar{e})=\alpha e$. Then $\alpha=\langle A(\bar{e}), \bar{e}\rangle=0$ which gives a contradiction and completes the proof. 
Lemma 2.2. Let $\mathfrak{g}$ be a nilpotent Lie algebra, $\mathfrak{a}$ and $\mathfrak{h}$, respectively, a Lie subalgebra of codimension one and an ideal of codimension two. Then $[\mathfrak{g}, \mathfrak{g}]$ is contained in $\mathfrak{a}$ and in $\mathfrak{h}$.

Proof. We have $\mathfrak{g} / \mathfrak{h}$ is a 2 -dimensional nilpotent Lie algebra and hence must be abelian. This implies that $[\mathfrak{g}, \mathfrak{g}] \subset \mathfrak{h}$. On the other hand, write $\mathfrak{g}=\mathfrak{a} \oplus \mathbb{R} y$. For any $x \in \mathfrak{a}$, we have

$$
[x, y]=a(x) y+u_{1}, \text { where } u_{1} \in \mathfrak{a} .
$$

Since $\mathfrak{a}$ is a Lie subalgebra then, for any $n \in \mathbb{N}^{*}, \operatorname{ad}_{x}^{n}(y)=a(x)^{n} y+u_{n}$ with $u_{n} \in \mathfrak{a}$. Since $\operatorname{ad}_{x}$ is nilpotent then $a(x)=0$ and the result follows.

We pursue with some general properties of flat pseudo-Euclidean Lie algebras. A pseudoEuclidean Lie algebra $(\mathfrak{g},\langle\rangle$,$) is a finite dimensional real Lie algebra \mathfrak{g}$ endowed with a non degenerate symmetric bilinear form $\langle$,$\rangle . We define a product (u, v) \mapsto u . v$ on $\mathfrak{g}$ called LeviCivita product by Koszul's formula

$$
2\langle u . v, w\rangle=\langle[u, v], w\rangle+\langle[w, u], v\rangle+\langle[w, v], u\rangle,
$$

for any $u, v, w \in \mathfrak{g}$. We denote by $\mathrm{L}_{u}: \mathfrak{g} \longrightarrow \mathfrak{g}$ and $\mathrm{R}_{u}: \mathfrak{g} \longrightarrow \mathfrak{g}$, respectively, the left multiplication and the right multiplication by $u$ given by $\mathrm{L}_{u} v=u \cdot v$ and $\mathrm{R}_{u} v=v \cdot u$. For any $u \in \mathfrak{g}$, $\mathrm{L}_{u}$ is skew-symmetric with respect to $\langle$,$\rangle and \operatorname{ad}_{u}=\mathrm{L}_{u}-\mathrm{R}_{u}$, where $\operatorname{ad}_{u}: \mathfrak{g} \longrightarrow \mathfrak{g}$ is given by $\operatorname{ad}_{u} v=[u, v]$. We call $(\mathfrak{g},\langle\rangle$,$) flat pseudo-Euclidean Lie algebra if the Levi-Civita product is$ left symmetric, i.e., for any $u, v, w \in \mathfrak{g}$,

$$
\operatorname{ass}(u, v, w)=\operatorname{ass}(v, u, w),
$$

where $\operatorname{ass}(u, v, w)=(u \cdot v) \cdot w-u \cdot(v \cdot w)$.

Remark 1. Let $G$ be a Lie group, and $\mu$ a left-invariant pseudo-Riemannian metric on $G$. Let $\mathfrak{g}=\operatorname{Lie}(G)$ and $\langle\rangle=,\mu_{e}$. Then the curvature of $(G, \mu)$ vanishes if and only if $(\mathfrak{g},\langle\rangle$,$) is a flat$ pseudo-Euclidean Lie algebra.

Let $(\mathfrak{g},\langle\rangle$,$) be a flat pseudo-Euclidean Lie algebra. The condition (2.2)$ is also equivalent to one of the following relations:

$$
\begin{aligned}
& \mathrm{L}_{[u, v]}=\left[\mathrm{L}_{u}, \mathrm{~L}_{v}\right], \\
& \mathrm{R}_{u, v}-\mathrm{R}_{v} \circ \mathrm{R}_{u}=\left[\mathrm{L}_{u}, \mathrm{R}_{v}\right],
\end{aligned}
$$

for any $u, v \in \mathfrak{g}$. We denote by $Z(\mathfrak{g})=\left\{u \in \mathfrak{g}, \operatorname{ad}_{u}=0\right\}$ the center of $\mathfrak{g}$. For any $u, v \in Z(\mathfrak{g})$ and $a, b \in \mathfrak{g}$, one can deduce easily from (2.1)-(2.4) that

$$
u . v=0, \mathrm{~L}_{u}=\mathrm{R}_{u}, \mathrm{~L}_{u} \circ \mathrm{L}_{v}=0 \text { and } u .(a . b)=a .(u . b) .
$$

Proposition 2.1. Let $(\mathfrak{g},\langle\rangle$,$) be a flat pseudo-Euclidean nilpotent non abelian Lie algebra. If$ $Z(\mathrm{~g})=\left\{u \in \mathfrak{g}, \mathrm{L}_{u}=\mathrm{R}_{u}=0\right\}$ then $Z(\mathrm{~g})$ is degenerate.

Proof. One can see easily that the orthogonal of the derived ideal of $g$ is given by

$$
[\mathfrak{g}, \mathfrak{g}]^{\perp}=\left\{u \in \mathfrak{g}, \mathbf{R}_{u}=\mathbf{R}_{u}^{*}\right\} .
$$

Then $Z(\mathfrak{g}) \subset[\mathfrak{g}, \mathfrak{g}]^{\perp}$ and hence $[\mathfrak{g}, \mathfrak{g}] \subset Z(\mathfrak{g})^{\perp}$. Since $\mathfrak{g}$ is nilpotent non abelian then $\{0\} \neq$ $[\mathfrak{g}, \mathfrak{g}] \cap Z(\mathfrak{g}) \subset Z(\mathfrak{g})^{\perp} \cap Z(\mathfrak{g})$. This shows that $Z(\mathfrak{g})$ is degenerate. 
Proposition 2.2. Let $(\mathfrak{g},\langle\rangle$,$) be a flat pseudo-Euclidean nilpotent Lie algebra. Then:$

1. If $(\mathfrak{g},\langle\rangle$,$) is Euclidean then \mathfrak{g}$ is abelian.

2. If $(\mathfrak{g},\langle\rangle$,$) is non abelian Lorentzian then Z(\mathfrak{g})$ is degenerate.

Proof. 1. According to (2.5), for any $u \in Z(\mathfrak{g}), \mathrm{L}_{u}$ is a nilpotent skew-symmetric endomorphism and hence must vanishes. This gives the result, by virtue of Proposition 2.1

2. This is a consequence of (2.5), Lemma 2.1 and Proposition 2.1.

Put $N(\mathfrak{g})=\bigcap_{u \in Z(\mathfrak{g})} \operatorname{ker} \mathrm{L}_{u}, \mathfrak{g}_{0}:=N(\mathfrak{g}) \cap Z(\mathfrak{g})^{\perp}$ and $\mathfrak{h}_{0}:=N(\mathfrak{g})^{\perp}$. These vector spaces and the following lemma which states their main properties will play a central role in this paper, namely, in the proof of Theorem 3.1

Lemma 2.3. Let $(\mathfrak{g},\langle\rangle$,$) be a flat pseudo-Euclidean nilpotent Lie algebra of signature (2, n-2)$, $n \geq 4$. Then:

1. $N(\mathfrak{g}), \mathfrak{g}_{0}$ and $\mathfrak{h}_{0}$ are left ideals for the Levi-Civita product, $\mathfrak{h}_{0} \subset \mathfrak{g}_{0}$, and $\mathfrak{h}_{0}$ is totally isotropic with $\operatorname{dim} \mathfrak{h}_{0} \leq 2$.

2. If $Z(\mathfrak{g})$ is non degenerate then the restriction of $\langle$,$\rangle to Z(\mathfrak{g})$ is positive definite, $\operatorname{dim} \mathfrak{b}_{0}=2$ and $\operatorname{dim}(Z(\mathfrak{g}) \cap[\mathfrak{g}, \mathfrak{g}])=1$. Moreover, if $z_{0}$ is a generator of $Z(\mathfrak{g}) \cap[\mathfrak{g}, \mathfrak{g}]$ with $\left\langle z_{0}, z_{0}\right\rangle=1$ then for any $u, v \in \mathfrak{g}$,

$$
[u, v]=[u, v]_{1}-2\left\langle\mathrm{~L}_{z_{0}} u, v\right\rangle z_{0},
$$

where $[u, v]_{1} \in Z(\mathfrak{g})^{\perp}$.

Proof. 1. Note first that, for any $u \in \mathfrak{g},\left(\operatorname{ker} \mathrm{L}_{u}\right)^{\perp}=\operatorname{ImL}_{u}$ and hence $\mathfrak{h}_{0}=\sum_{u \in Z(\mathfrak{g})} \operatorname{ImL}_{u}$. From (2.5), we have clearly that $Z(\mathfrak{g}) \subset N(\mathfrak{g})$ and, for any $u, v \in Z(\mathfrak{g}), \operatorname{ImL}_{u} \subset \operatorname{ker}_{v}$. Thus $\mathfrak{h}_{0} \subset \mathfrak{g}_{0}$. This implies that $\mathfrak{h}_{0}$ is totally isotropic and since the signature is $(2, n-2)$ one must have $\operatorname{dim} \mathfrak{h}_{0} \leq 2$. One can deduce easily from the third relation in (2.5) that $N(\mathfrak{g})$ is a left ideal. This implies, since the left multiplication are skew-symmetric that $\mathfrak{h}_{0}$ and $\mathfrak{g}_{0}$ are also left ideals.

2. Suppose now that $Z(\mathfrak{g})$ is non degenerate. If $\operatorname{dim} \mathfrak{h}_{0} \leq 1$ then, according to Lemma 2.1 $\mathrm{L}_{u}=0$ for any $u \in Z(\mathfrak{g})$ and hence, by virtue of Proposition $2.1, Z(\mathfrak{g})$ is degenerate. So we must have $\operatorname{dim} \mathfrak{h}_{0}=2$ and the restriction of $\langle$,$\rangle to Z(\mathfrak{g})^{\perp}$ is of signature $\left(2, \operatorname{dim} Z(\mathfrak{g})^{\perp}-2\right)$ which implies that the restriction of $\langle$,$\rangle to Z(\mathfrak{g})$ is definite positive. On the other hand, according to what above we can choose two vectors $\left(\bar{e}_{1}, \bar{e}_{2}\right)$ of $Z(\mathfrak{g})^{\perp}$ such that $Z(\mathfrak{g})^{\perp}=$ $\mathfrak{g}_{0} \oplus \operatorname{Span}\left\{\bar{e}_{1}, \bar{e}_{2}\right\}$. So,

$$
[\mathfrak{g}, \mathfrak{g}]=\left[Z(\mathfrak{g})^{\perp}, Z(\mathfrak{g})^{\perp}\right]=\mathbb{R}\left[\bar{e}_{1}, \bar{e}_{2}\right]+\left[\bar{e}_{1}, \mathfrak{g}_{0}\right]+\left[\bar{e}_{2}, \mathfrak{g}_{0}\right]+\left[\mathfrak{g}_{0}, \mathfrak{g}_{0}\right] .
$$

We have that $\mathfrak{g}_{0}$ is a left ideal for the Levi-Civita product and for any $a \in \mathfrak{g}_{0}, b \in \mathfrak{g}$ and $u \in Z(\mathfrak{g})$

$$
\langle a . b, u\rangle=-\langle b, a . u\rangle=-\langle b, u \cdot a\rangle=0
$$

and hence $\mathfrak{g}_{0} \cdot \mathfrak{g} \subset Z(\mathfrak{g})^{\perp}$. This implies that $\left[\bar{e}_{1}, \mathfrak{g}_{0}\right]+\left[\bar{e}_{2}, \mathfrak{g}_{0}\right]+\left[\mathfrak{g}_{0}, \mathfrak{g}_{0}\right] \subset Z(\mathfrak{g})^{\perp}$. Moreover, $\left[\bar{e}_{1}, \bar{e}_{2}\right]=z+v_{0}$, where $z \in Z(\mathfrak{g}), z \neq 0$ since $Z(\mathfrak{g}) \cap[\mathfrak{g}, \mathfrak{g}] \neq 0$ and $v_{0} \in Z(\mathfrak{g})^{\perp}$. So $[\mathfrak{g}, \mathfrak{g}]=\mathbb{R} z \oplus F$ where $F$ is a vector subspace of $Z(\mathfrak{g})^{\perp}$. From this relation, we can deduce that $Z(\mathfrak{g}) \cap[\mathfrak{g}, \mathfrak{g}]=\mathbb{R} z$ and (2.7) follows immediately. 


\section{The center of a flat pseudo-Euclidean nilpotent Lie algebra of signature $(2, n-2)$ is} degenerate

The purpose of this section is to prove the following theorem.

Theorem 3.1. Let $(\mathfrak{g},\langle\rangle$,$) be a flat pseudo-Euclidean nilpotent non abelian Lie algebra of$ signature $(2, n-2)$ with $n \geq 4$. Then $Z(\mathfrak{g})$ is degenerate.

Proof. We proceed by contradiction and we suppose that $Z(\mathfrak{g})$ is non degenerate, i.e., $\mathfrak{g}=Z(\mathfrak{g}) \oplus$ $Z(\mathfrak{g})^{\perp}$. As in Lemma 2.3, we consider $\mathfrak{g}_{0}=\left\{v \in Z(\mathfrak{g})^{\perp} / \mathrm{L}_{u} v=0, \forall u \in Z(\mathfrak{g})\right\}$ and $\mathfrak{h}_{0}$ its orthogonal in $Z(\mathfrak{g})^{\perp}$. We have both $\mathfrak{h}_{0}$ and $\mathfrak{g}_{0}$ are left ideals for the Levi-Civita product, $\mathfrak{h}_{0} \subset \mathfrak{g}_{0}$ and $\mathfrak{h}_{0}$ is totally isotropic of dimension 2. Moreover, if $z_{0}$ is a unit generator of $Z(\mathfrak{g}) \cap[\mathfrak{g}, \mathfrak{g}]$ then, for any $u, v \in \mathfrak{g}$,

$$
[u, v]=[u, v]_{1}-2\left\langle\mathrm{~L}_{z_{0}} u, v\right\rangle z_{0},
$$

where $[u, v]_{1} \in Z(\mathfrak{g})^{\perp}$. This relation shows that $\mathrm{L}_{z_{0}} \neq 0$ and since $\mathrm{L}_{z_{0}}^{2}=0$ and $\operatorname{ImL}_{z_{0}} \subset \mathfrak{h}_{0}$, by virtue of Lemma 2.1, $\operatorname{ImL}_{z_{0}}=\mathfrak{h}_{0}$ and $\operatorname{ker} \mathrm{L}_{z_{0}}=Z(\mathfrak{g}) \oplus \mathfrak{g}_{0}$. Moreover, from (3.1), one can check easily that $[,]_{1}$ satisfies Jacobi identity and $\left(Z(\mathfrak{g})^{\perp},[,]_{1}\right)$ becomes a nilpotent Lie algebra. We denote by $\circ$ the Levi-Civita product of $\left(Z(\mathfrak{g})^{\perp},[,]_{1},\langle\rangle,\right)$ and we have obviously, for any $u, v \in Z(\mathfrak{g})^{\perp}$,

$$
u . v=u \circ v-\left\langle\mathrm{L}_{z_{0}} u, v\right\rangle z_{0} .
$$

Let $C(\mathfrak{g})$ denote the center of $\left(Z(\mathfrak{g})^{\perp},[,]_{1}\right)$. We have $C(\mathfrak{g}) \neq 0$ and $C(\mathfrak{g}) \cap \mathfrak{g}_{0}=\{0\}$. Indeed, if $u \in C(\mathfrak{g}) \cap \mathfrak{g}_{0}$, then for any $v \in Z(\mathfrak{g})^{\perp}$,

$$
[u, v]=[u, v]_{1}-2\left\langle\mathrm{~L}_{z_{0}} u, v\right\rangle z_{0}=0,
$$

hence $u \in Z(\mathfrak{g})$ and then $u=0$. This implies that $1 \leq \operatorname{dim} C(\mathfrak{g}) \leq 2$ and for any $u \in C(\mathfrak{g}) \backslash\{0\}$, $z_{0} . u \neq 0$.

Let $z$ be a non-null vector in $C(\mathfrak{g})$ then $z_{0} \cdot z$ is a non-null vector in $\mathfrak{h}_{0}$. From (2.3) we get $\mathrm{L}_{z} \circ \mathrm{L}_{z_{0}}=\mathrm{L}_{z_{0}} \circ \mathrm{L}_{z}$ and by using (2.4) we have

$$
\mathrm{R}_{z . z_{0}}=\mathrm{R}_{z_{0}} \circ \mathrm{R}_{z}=\mathrm{L}_{z_{0}} \circ \mathrm{R}_{z} .
$$

For any $u \in Z(\mathfrak{g})^{\perp}$, we have from (3.2) and the fact that $z \in C(\mathfrak{g})$,

$$
\mathrm{L}_{z} u=z \circ u-\left\langle z_{0} . z, u\right\rangle z_{0} \quad \text { and } \quad \mathrm{R}_{z} u=u \circ z+\left\langle z_{0} . z, u\right\rangle z_{0}=z \circ u+\left\langle z_{0} . z, u\right\rangle z_{0} .
$$

Thus $\mathrm{L}_{z} u=\mathrm{R}_{z} u-2\left\langle z_{0} . z, u\right\rangle z_{0}$. This relation is also true for $u \in Z(\mathrm{~g})$ since $z_{0} \cdot u=0$ and hence $\mathrm{L}_{z}=\mathrm{R}_{z}+A_{z}$, where $\mathrm{A}_{z}=-2\left\langle z . z_{0},.\right\rangle z_{0}$. Since $\mathrm{L}_{z_{0}} \circ A_{z}=0$, we deduce that

$$
\mathrm{R}_{z . z_{0}}=\mathrm{L}_{z_{0}} \circ \mathrm{R}_{z}=\mathrm{L}_{z_{0}} \circ\left(\mathrm{L}_{z}-A_{z}\right)=\mathrm{L}_{z_{0}} \circ \mathrm{L}_{z}=\mathrm{L}_{z} \circ \mathrm{L}_{z_{0}}
$$

This relation implies that $\mathrm{R}_{z \cdot z_{0}}$ is symmetric and $\mathfrak{g}_{0} \oplus Z(\mathfrak{g}) \subset \operatorname{ker} \mathrm{R}_{z \cdot z_{0}}$. From (3.2), we have $z . z=0$, and hence $\mathfrak{g}_{0} \oplus \mathbb{R} z \oplus Z(\mathfrak{g}) \subset \operatorname{ker} \mathrm{R}_{z . z_{0}}$. From the symmetry of $\mathrm{R}_{z . z_{0}}$ we deduce that $\operatorname{ImR}_{z . z_{0}}=\left(\operatorname{ker} \mathrm{R}_{z . z_{0}}\right)^{\perp}$ and finally $\operatorname{ImR}_{z . z_{0}} \subset\left(\mathfrak{g}_{0} \oplus \mathbb{R} z \oplus Z(\mathfrak{g})\right)^{\perp}=\mathbb{R} z . z_{0}$. So we can write, for any $u \in \mathfrak{g}$,

$$
\mathrm{R}_{z \cdot z_{0}}(u)=a_{1}(u) z \cdot z_{0}=\alpha\left\langle z \cdot z_{0}, u\right\rangle z \cdot z_{0},
$$

where $a_{1} \in \mathfrak{g}^{*}$ and $\alpha \in \mathbb{R}$. We will show now that $\mathrm{R}_{z . z_{0}}=0$. 
Put $e_{1}=z_{0} . z$. Since the orthogonal of $z$ in $Z(\mathfrak{g})^{\perp}$ is different from the orthogonal of $e_{1}$ in $Z(\mathfrak{g})^{\perp}$, we can choose $\bar{z} \in Z(\mathfrak{g})^{\perp}$ such that $\langle z, \bar{z}\rangle=0$ and $\left\langle e_{1}, \bar{z}\right\rangle=1$. We put $e_{2}=-z_{0} \cdot \bar{z}$. We have $\left\langle e_{2}, z\right\rangle=1, Z(\mathfrak{g})^{\perp}=\mathfrak{g}_{0} \oplus \operatorname{span}\{z, \bar{z}\}$ and $\left(e_{1}, e_{2}\right)$ is a basis of $\mathfrak{h}_{0}$. Now $\mathfrak{h}_{0}$ is a 2-dimensional subalgebra of a nilpotent Lie algebra then it must be abelian and since $\mathfrak{h}_{0} \subset$ ker $\mathrm{R}_{e_{1}}$ we deduce that $e_{1} \cdot e_{1}=e_{1} \cdot e_{2}=e_{2} . e_{1}=0$. Moreover, $\mathfrak{h}_{0}$ is a left ideal and we can write, for any $u \in \mathfrak{g}$,

$$
u . e_{1}=a_{1}(u) e_{1} \quad \text { and } \quad u . e_{2}=a_{2}(u) e_{1}+b_{2}(u) e_{2} .
$$

From the relation $u .\left(z_{0} \cdot z\right)=z_{0} .(u . z)$ shown in $(2.5)$, we deduce that $a_{1}(u) z_{0} \cdot z=z_{0} \cdot(u . z), a_{1}(u) z-$ $u . z \in \operatorname{ker} \mathrm{L}_{z_{0}}=\mathfrak{h}_{0}^{\perp}$ and hence

$$
0=a_{1}(u)\left\langle z, e_{2}\right\rangle-\left\langle u . z, e_{2}\right\rangle=a_{1}(u)\left\langle z, e_{2}\right\rangle+\left\langle z, u . e_{2}\right\rangle=a_{1}(u)+b_{2}(u) .
$$

Thus $b_{2}=-a_{1}$. Using the fact that the curvature vanishes, we get

$$
\begin{aligned}
{[u, v] . e_{2} } & =u \cdot\left(v \cdot e_{2}\right)-v \cdot\left(u \cdot e_{2}\right) \\
& =u \cdot\left(a_{2}(v) e_{1}-a_{1}(v) e_{2}\right)-v \cdot\left(a_{2}(u) e_{1}-a_{1}(u) e_{2}\right) \\
& =2\left(a_{2}(v) a_{1}(u)-a_{1}(v) a_{2}(u)\right) e_{1} .
\end{aligned}
$$

Thus

$$
a_{2}([u, v])=2\left(a_{2}(v) a_{1}(u)-a_{1}(v) a_{2}(u)\right) .
$$

By taking $u=z$ and $v=\bar{z}$ in this relation and since $a_{2}\left(z_{0}\right)=0, a_{1}(z)=0$ and, by virtue of (3.1), $[z, \bar{z}]=-2 z_{0}$, we get $a_{2}(z) a_{1}(\bar{z})=0$. Now

$$
a_{1}(\bar{z}) e_{1}=\mathrm{R}_{e_{1}}(\bar{z}) \stackrel{3.3}{=} \mathrm{L}_{z} \circ \mathrm{L}_{z 0}(\bar{z})=-z . e_{2}=-a_{2}(z) e_{1} .
$$

This relation and $a_{2}(z) a_{1}(\bar{z})=0$ imply that $\mathrm{R}_{e_{1}}(\bar{z})=0$. But $\mathfrak{g}_{0} \oplus \mathbb{R} z \oplus Z(\mathfrak{g}) \subset$ ker $\mathrm{R}_{e_{1}}$ so finally $\mathrm{R}_{e_{1}}=0$. To complete, we will show that $e_{1} \in Z(\mathfrak{g})$, i.e, $\mathrm{L}_{e_{1}}=\operatorname{ad}_{e_{1}}=0$ and we will get a contradiction.

Note first that $\mathrm{L}_{e_{1}}$ is nilpotent, $\mathrm{L}_{e_{1}}\left(\mathfrak{h}_{0}\right)=0$ and $\mathrm{L}_{e_{1}}\left(\mathfrak{g}_{0}\right) \subset \mathfrak{g}_{0}$. So $\mathrm{L}_{e_{1}}$ induces on the Euclidean vector space $\mathfrak{g}_{0} / \mathfrak{h}_{0}$ a skew-symmetric nilpotent endomorphism which must then vanish. So $\mathrm{L}_{e_{1}}\left(\mathfrak{g}_{0}\right) \subset \mathfrak{h}_{0}$. On the other hand, by virtue of $\left[3.1, e_{1} . z=\left[e_{1}, z\right]=0\right.$. So for any $x \in \mathfrak{g}_{0}$, $e_{1} \cdot x=\left[e_{1}, x\right]=a(x) e_{1}+b(x) e_{2}$. This implies that $b(x)=\left\langle e_{1} \cdot x, z\right\rangle=-\left\langle x, e_{1} \cdot z\right\rangle=0$. But $\operatorname{ad}_{x}$ is nilpotent so $a(x)=0$ and we deduce that $\mathrm{L}_{e_{1}}\left(\mathfrak{g}_{0}\right)=0$. So far, we have shown that $\mathfrak{g}_{0} \oplus \mathbb{R} z \oplus Z(\mathfrak{g}) \subset \operatorname{ker} \mathrm{L}_{e_{1}}$ and hence its image has a dimension less or equal to 1 . Moreover, $\operatorname{ImL}_{e_{1}} \subset \mathfrak{h}_{0}$ and hence $\mathrm{L}_{e_{1}}^{2}=0$ and we can conclude by using Lemma 2.1

\section{Flat pseudo-Euclidean nilpotent Lie algebras of signature $(2, n-2)$ are obtained by the double extension process}

In this section, based on Theorem 3.1, we will show that any flat pseudo-Euclidean nilpotent Lie algebra of signature $(2, n-2)$ can be obtained by the double extension process from a Lorentzian or an Euclidean flat nilpotent Lie algebra. To do so we need first to recall the double extension process introduced by Aubert and Medina [2]. Note that Propositions 3.1 and 3.2 in the paper [2] are essential in this process.

Let $\left(B,[,]_{0},\langle,\rangle_{0}\right)$ be a pseudo-Riemannian flat Lie algebra, $\xi, D: B \longrightarrow B$ two endomorphisms of $B, b_{0} \in B$ and $\mu \in \mathbb{R}$ such that: 
1. $\xi$ is a 1 -cocycle of $\left(B,[,]_{0}\right)$ with respect to the representation $\mathrm{L}: B \longrightarrow \operatorname{End}(B)$ defined by the left multiplication associated to the Levi-Civita product, i.e., for any $a, b \in B$,

$$
\xi([a, b])=\mathrm{L}_{a} \xi(b)-\mathrm{L}_{b} \xi(a)
$$

2. $D-\xi$ is skew-symmetric with respect to $\langle,\rangle_{0}$,

$$
[D, \xi]=\xi^{2}-\mu \xi-\mathrm{R}_{b_{0}},
$$

and for any $a, b \in B$

$$
a \cdot \xi(b)-\xi(a \cdot b)=D(a) \cdot b+a \cdot D(b)-D(a \cdot b) .
$$

We call $\left(\xi, D, \mu, b_{0}\right)$ satisfying the two conditions above admissible.

Given $\left(\xi, D, \mu, b_{0}\right)$ admissible, we endow the vector space $\mathfrak{g}=\mathbb{R} e \oplus B \oplus \mathbb{R} \bar{e}$ with the inner product $\langle$,$\rangle which extends \langle,\rangle_{0}$, for which $\operatorname{span}\{e, \bar{e}\}$ and $B$ are orthogonal, $\langle e, e\rangle=\langle\bar{e}, \bar{e}\rangle=0$ and $\langle e, \bar{e}\rangle=1$. We define also on $g$ the bracket

$$
[\bar{e}, e]=\mu e,[\bar{e}, a]=D(a)-\left\langle b_{0}, a\right\rangle_{0} e \quad \text { and } \quad[a, b]=[a, b]_{0}+\left\langle\left(\xi-\xi^{*}\right)(a), b\right\rangle_{0} e,
$$

where $a, b \in B$ and $\xi^{*}$ is the adjoint of $\xi$ with respect to $\langle,\rangle_{0}$. Then $(\mathfrak{g},[],,\langle\rangle$,$) is a flat pseudo-$ Euclidean Lie algebra called double extension of $\left(B,[,]_{0},\langle,\rangle_{0}\right)$ according to $\left(\xi, D, \mu, b_{0}\right)$. Using this method, Aubert and Medina characterize a flat Lorentzian nilpotent Lie algebras. They show that $(\mathfrak{g},\langle\rangle$,$) is a flat Lorentzian nilpotent Lie algebra if and only if (\mathfrak{g},\langle\rangle$,$) is a double extension$ of an Euclidean abelian Lie algebra according to $\mu=0, D=\xi$ and $b_{0}$ where $D^{2}=0$.

Theorem 4.1. Let $(\mathfrak{g},\langle\rangle$,$) be a flat pseudo-Euclidean nilpotent Lie algebra of signature (2, n-$ 2). Then, for any $e \in Z(\mathfrak{g}) \cap Z(\mathfrak{g})^{\perp}, \mathrm{L}_{e}=\mathrm{R}_{e}=0$. Moreover, $Z(\mathfrak{g})+Z(\mathfrak{g})^{\perp}$ is a two-sided ideal with respect to the Levi-Civita product.

Proof. Recall that $[\mathfrak{g}, \mathfrak{g}]^{\perp}=\left\{u \in \mathfrak{g}, \mathrm{R}_{u}=\mathrm{R}_{u}^{*}\right\}$, put $\mathfrak{a}=Z(\mathfrak{g})+Z(\mathfrak{g})^{\perp}$ and consider $N(\mathfrak{g})=\{v \in$ $\left.\mathfrak{g} / \mathrm{L}_{u} v=0, \forall u \in Z(\mathfrak{g})\right\}$ and $\mathfrak{h}_{0}$ its orthogonal. We have seen in Lemma 2.3 that both $N(\mathfrak{g})$ and $\mathfrak{h}_{0}$ are left ideals and $\mathfrak{h}_{0}$ is totally isotropic. We have seen that if $\operatorname{dim} \mathfrak{h}_{0} \leq 1$ then $N(\mathfrak{g})=\mathfrak{g}$ and hence any vector $e \in Z(\mathfrak{g}) \cap Z(\mathfrak{g})^{\perp}$ satisfies the conditions required. Suppose that $\operatorname{dim} \mathfrak{h}_{0}=2$. We claim that $Z(\mathfrak{g}) \cap Z(\mathfrak{g})^{\perp} \subset \mathfrak{h}_{0}$. This is a consequence of the fact that $Z(\mathfrak{g}) \cap Z(\mathfrak{g})^{\perp} \subset Z(\mathfrak{g}) \subset N(\mathfrak{g})$ and the fact that $N(\mathfrak{g}) / \mathfrak{h}_{0}$ is Euclidean. We distinguish two cases:

1. $Z(\mathfrak{g}) \cap Z(\mathfrak{g})^{\perp}=\mathfrak{h}_{0}$ and hence $\mathfrak{a}=N(\mathfrak{g})$. We have that $\mathfrak{g} \cdot N(\mathfrak{g}) \subset N(\mathfrak{g})$ and for any $u \in N(\mathfrak{g})$, $w \in \mathfrak{g}$ and $v \in \mathfrak{h}_{0}, v \cdot u=u . v=0$ and hence $\langle u . w, v\rangle=0$. This implies that $N(\mathfrak{g})$ is an ideal for the Lie bracket and, according to Lemma 2.2, [g, g] $\subset N(\mathfrak{g})$. We deduce that $Z(\mathfrak{g}) \cap Z(\mathfrak{g})^{\perp} \subset[\mathfrak{g}, \mathfrak{g}]^{\perp}$ and hence for any $e \in Z(\mathfrak{g}) \cap Z(\mathfrak{g})^{\perp}, \mathrm{L}_{e}$ is both skew-symmetric and symmetric and hence $\mathrm{L}_{e}=\mathrm{R}_{e}=0$.

2. $\operatorname{dim} Z(\mathfrak{g}) \cap Z(\mathfrak{g})^{\perp}=1$. Since $\left.Z(\mathfrak{g}) \cap Z(\mathfrak{g})^{\perp} \subset \mathfrak{h}\right)_{0}$, we have $N(\mathfrak{g}) \subset \mathfrak{a}$ and $\mathfrak{a}=\mathfrak{g}_{0}+\mathbb{R} y$. We have $\mathfrak{g} . \mathfrak{g}_{0} \subset \mathfrak{a}$ and for any $u \in \mathfrak{g}_{0}, w \in \mathfrak{g}$ and $v \in Z(\mathfrak{g}) \cap Z(\mathfrak{g})^{\perp}, v . u=u . v=0$ and hence $\langle u . w, v\rangle=0$. Thus $N(\mathfrak{g}) \cdot \mathfrak{g} \subset \mathfrak{a}$. Moreover, for any $v \in Z(\mathfrak{g}) \cap Z(\mathfrak{g})^{\perp},\langle y \cdot y, v\rangle=0$ and then $y . y \in \mathfrak{a}$. In particular, $\mathfrak{a} \cdot \mathfrak{a} \subset \mathfrak{a}$ and hence $\mathfrak{a}$ is a subalgebra. According to Lemma 2.2 , $[\mathfrak{g}, \mathfrak{g}] \subset \mathfrak{a}$ and hence $Z(\mathfrak{g}) \cap Z(\mathfrak{g})^{\perp} \subset[\mathfrak{g}, \mathfrak{g}]^{\perp}$. This implies that for any $e \in Z(\mathfrak{g}) \cap Z(\mathfrak{g})^{\perp}$, $\mathrm{L}_{e}=\mathrm{R}_{e}=0$ and $\mathfrak{a}$ is a two-sided ideal.

Theorem 4.2. Let $(\mathfrak{g},\langle\rangle$,$) be a flat pseudo-Euclidean nilpotent Lie algebra of signature (2, n-$ 2). Then $(\mathfrak{g},\langle\rangle$,$) is a double extension of a flat Lorentzian nilpotent Lie algebra, according to$ $\mu=0, D$, $\xi$ and $b_{0}$ where $D$ is a nilpotent endomorphism. 
Proof. Let $e$ be a non-null vector in $Z(\mathfrak{g}) \cap Z(\mathfrak{g})^{\perp}$ and put $\mathrm{I}=\mathbb{R} e$. According to Theorem 4.1, $\mathrm{I}$ is a totally isotropic two-sided ideal with respect to the Levi-Civita product. Moreover, $\mathrm{I}^{\perp}$ is also a two sided ideal. Then, according to [2],$(\mathfrak{g},\langle\rangle$,$) is a double extension of flat Lorentzian$ Lie algebra $\left(B,\langle,\rangle_{B}\right)$. From (4.4) and the fact that $g$ is nilpotent we deduce that $D$ is a nilpotent endomorphism, and $B$ is a nilpotent Lie algebra.

Remark 2. According to [2], flat Lorentzian nilpotent Lie algebra are double extension of abelian Euclidean Lie algebras. Then flat pseudo-Euclidean nilpotent Lie algebras of signature $(2, n-2)$ are obtained by applying twice the double extension process, starting from abelian Euclidean Lie algebras.

Example 1. Let $(\mathfrak{g},\langle\rangle$,$) be a 4-dimensional flat pseudo-Euclidean nilpotent Lie algebras of$ signature (2,2). According to theorem 4.2 ( $\mathfrak{g},\langle\rangle$,$) is a double extension of a 2-dimensional$ abelian Lorentzian Lie algebra $\left(B,\langle,\rangle_{B}\right)$ with $D^{2}=0$. The conditions (4.1)-(4.3) are equivalent to $[D, \xi]=\xi^{2}$ and $D-\xi$ is skew-symmetric, which implies that $D=\xi$. Then there exists a basis $\left\{e_{1}, e_{2}\right\}$ of $B$ such that the matrix of $D$ in this basis has the form

$$
\left(\begin{array}{ll}
0 & \alpha \\
0 & 0
\end{array}\right) \text {, where } \alpha \in \mathbb{R} \text {. }
$$

Let $\langle,\rangle_{B}$ be any Lorentzian metric in B. Then according to (4.4), $\mathfrak{g}=\operatorname{span}\left\{\bar{e}, e, e_{1}, e_{2}\right\}$ with the non vanishing Lie brackets

$$
\left[\bar{e}, e_{1}\right]=\beta e,\left[\bar{e}, e_{2}\right]=\alpha e_{1}+\gamma e,\left[e_{1}, e_{2}\right]=\delta e, \text { where } \alpha, \beta, \gamma, \delta \in \mathbb{R},
$$

and the metric in $\mathfrak{g}$ is an extension orthogonal of $\langle,\rangle_{B}$ such that $\langle\bar{e}, \bar{e}\rangle=\langle e, e\rangle=0$ and $\langle\bar{e}, e\rangle=1$. It is easy to show that $\mathfrak{g}$ is isomorphic to one of the following Lie algebras:

- $\mathbb{R}^{4}$ : The 4-dimensional abelian Lie algebra (if $\alpha=\beta=\gamma=\delta=0$ ).

- $\mathcal{H}_{3} \oplus \mathbb{R}$ : The extension trivial of $\mathcal{H}_{3}$ (if $\alpha=0$ and $(\beta, \gamma) \neq(0,0)$ or $\alpha \neq 0$ and $\beta=\delta=0$ ).

- The 4-dimensional filiform Lie algebra: $\left[\bar{e}, e_{1}\right]=e,\left[\bar{e}, e_{2}\right]=e_{1}($ If $\alpha \neq 0$ and $(\beta, \delta) \neq$ $(0,0))$.

\section{Flat pseudo-Euclidean 2-step nilpotent Lie algebras}

A 2-step nilpotent Lie algebra is a non-abelian Lie algebra $\mathfrak{g}$ which satisfies $[\mathfrak{g}, \mathfrak{g}] \subset Z(\mathfrak{g})$. Let $(\mathfrak{g},\langle\rangle$,$) be a flat pseudo-Euclidean 2-step nilpotent Lie algebra. In [7], the author showed that if$ the metric $\langle$,$\rangle is Lorentzian, then \mathrm{g}$ is an extension trivial of $\mathcal{H}_{3}$, where $\mathcal{H}_{3}$ is a 3 -dimensional Heisenberg Lie algebra. Let us studies some properties of $(\mathfrak{g},\langle\rangle$,$) in other signatures.$

We consider $N(\mathfrak{g})=\bigcap_{u \in Z(\mathfrak{g})} \operatorname{ker} \mathrm{L}_{u}$, and $\mathfrak{h}_{0}:=N(\mathfrak{g})^{\perp}$. According to Lemma2.3 $\mathfrak{h}_{0} \subset N(\mathfrak{g})$. If $N(\mathfrak{g}) \neq \mathfrak{g}$, then $N(\mathfrak{g})$ is degenerate. On the other hand, for any $z \in Z(\mathfrak{g}), a \in N(\mathfrak{g})$ and $u \in \mathfrak{g}$ we have

$$
\langle u . a, z\rangle=-\langle a, z . u\rangle=\langle z . a, u\rangle=-\langle a . u, z\rangle=0 .
$$

This implies that $\mathfrak{g} \cdot N(\mathfrak{g}) \subset Z(\mathfrak{g})^{\perp}$ and $N(\mathfrak{g}) \cdot \mathfrak{g} \subset Z(\mathfrak{g})^{\perp}$. Thus

$$
[\mathfrak{g}, N(\mathfrak{g})] \subset Z(\mathfrak{g}) \cap Z(\mathfrak{g})^{\perp} .
$$

Proposition 5.1. Let $(\mathfrak{g},\langle\rangle$,$) be a flat pseudo-Euclidean 2-step nilpotent Lie algebra. Then$ 
1. $Z(\mathfrak{g})$ is degenerate.

2. For any $e \in Z(\mathfrak{g}) \cap Z(\mathfrak{g})^{\perp}, \mathrm{L}_{e}=\mathrm{R}_{e}=0$.

3. For any $x, y \in Z(\mathfrak{g})^{\perp},\langle[x, y],[x, y]\rangle=0$.

Proof. $\quad$ 1. Suppose that $Z(\mathfrak{g})$ is non degenerate, i.e., $Z(\mathfrak{g}) \cap Z(\mathfrak{g})^{\perp}=\{0\}$.

- If $\mathfrak{g}=N(\mathfrak{g})$ then according to $5.1,[\mathfrak{g}, \mathfrak{g}]=0$ which is impossible.

- If $\mathfrak{g} \neq N(\mathfrak{g})$ then $[\mathfrak{g}, N(\mathfrak{g})]=0$ and hence $N(\mathfrak{g})=Z(\mathfrak{g})$ which is impossible since $N(\mathfrak{g})$ is degenerate.

2. Let $e \in Z(\mathfrak{g}) \cap Z(\mathfrak{g})^{\perp}$. Since $Z(\mathfrak{g})^{\perp} \subset[\mathfrak{g}, \mathfrak{g}]^{\perp}$, then according to (2.6), $\mathrm{L}_{e}=\mathrm{R}_{e}$ is both symmetric and skew-symmetric and hence must vanish.

3. According to (2.1), we have for any $x, y \in Z(\mathfrak{g})^{\perp} x \cdot y=\frac{1}{2}[x, y]$. Using (2.3), we have $[x, y] \cdot x=x .(y \cdot x)-y \cdot(x . x)$, then $[x, y] \cdot x=0$. In particular $\langle[x, y] . x, y\rangle=0$. Since $\mathrm{L}_{x}$ is skew-symmetric, thus $\langle[x, y],[x, y]\rangle=0$.

Proposition 5.2. Let $(\mathfrak{g},\langle\rangle$,$) be a flat pseudo-Euclidean 2-step nilpotent Lie algebra. Then$ $(\mathfrak{g},\langle\rangle$,$) is obtained by a sequence of double extension, starting from an abelian pseudo-$ Euclidean Lie algebra.

Proof. Let e be a non-null vector in $Z(\mathfrak{g}) \cap Z(\mathfrak{g})^{\perp}$. Since $\mathrm{L}_{e}=\mathrm{R}_{e}=0$, then $\mathrm{I}=\mathbb{R} e$ is a totally isotropic two sided ideal, and $\mathrm{I}^{\perp}$ is also a two sided ideal. Thus, $(\mathfrak{g},\langle\rangle$,$) is a double extension$ of a pseudo-Euclidean Lie algebra $\left(B_{1},\langle,\rangle_{1}\right)$. According to (4.4), $B_{1}$ is either abelian or 2-step nilpotent. If $B_{1}$ is 2 -step nilpotent, then it's also a double extension of $\left(B_{2},\langle,\rangle_{2}\right)$. Since a 2-step nilpotent Lie algebra can not admit a flat Euclidean metric, then there exists $k \in \mathbb{N}^{*}$ such that $B_{k}$ is abelian.

Proposition 5.3. Let $(\mathfrak{g},\langle\rangle$,$) be a flat pseudo-Euclidean 2-step nilpotent Lie algebra of signa-$ ture $(p, p+q)$. If $\operatorname{dim}\left(Z(\mathfrak{g}) \cap Z(\mathfrak{g})^{\perp}\right)=p$ then $Z(\mathfrak{g})^{\perp}$ is abelian.

Proof. Let $\left\{e_{1}, \ldots, e_{p}\right\}$ be a basis of $Z(\mathfrak{g}) \cap Z(\mathfrak{g})^{\perp}$, then we can whrite $Z(\mathfrak{g})=Z_{1} \oplus \operatorname{span}\left\{e_{1}, \ldots, e_{p}\right\}$ where $\left(Z_{1},\langle,\rangle_{Z_{1} \times Z_{1}}\right)$ is euclidean. In $Z_{1}^{\perp}$ we can choose a totaly isotropic subspace $\operatorname{span}\left\{\bar{e}_{1}, \ldots, \bar{e}_{p}\right\}$ such that, $\left\langle e_{i}, \bar{e}_{j}\right\rangle=0$ for $i \neq j$, and $\left\langle e_{i}, \bar{e}_{i}\right\rangle=1$. Let $B_{1}$ be the orthogonal of $Z_{1} \oplus \operatorname{span}\left\{e_{1}, \ldots, e_{p}\right\} \oplus$ $\operatorname{span}\left\{\bar{e}_{1}, \ldots, \bar{e}_{p}\right\}$. Thus we get a decomposition

$$
\mathfrak{g}=Z_{1} \oplus \operatorname{span}\left\{e_{1}, \ldots, e_{p}\right\} \oplus B_{1} \oplus \operatorname{span}\left\{\bar{e}_{1}, \ldots, \bar{e}_{p}\right\} .
$$

We have $Z(\mathfrak{g})^{\perp}=B_{1} \oplus \operatorname{span}\left\{\bar{e}_{1}, \ldots, \bar{e}_{p}\right\}$, and $\left(B_{1},\langle,\rangle_{B_{B_{1} \times B_{1}}}\right)$ is euclidean. Let $x, y \in Z(\mathfrak{g})^{\perp}, z \in Z(\mathfrak{g})$ and $k \in\{1, \ldots, p\}$. We have $\langle[x, y],[x, y]\rangle=0, z . \bar{e}_{k} \in Z(\mathfrak{g})^{\perp}$ and $\left\langle z . \bar{e}_{k}, z . \bar{e}_{k}\right\rangle=0$. Since $Z_{1}$ and $B_{1}$ are euclidean, then $[x, y]$ and $z . \bar{e}_{k}$ are in $Z(\mathfrak{g}) \cap Z(\mathfrak{g})^{\perp}$. Thus

$$
0=\left\langle z . \bar{e}_{k}, x\right\rangle=-\frac{1}{2}\left\langle\left[\bar{e}_{k}, x\right], z\right\rangle,
$$

which implies that $\left[\bar{e}_{k}, x\right] \in Z(\mathfrak{g}) \cap Z(\mathfrak{g})^{\perp}$. Using the flatness of the metric, then $\left[\bar{e}_{k}, x\right] . x=$ $\bar{e}_{k} \cdot(x \cdot x)-x \cdot\left(\bar{e}_{k} \cdot x\right)$, thus $x \cdot\left(\bar{e}_{k} \cdot x\right)=0$. Let $\left\{b_{1}, \ldots, b_{r}\right\}$ be an orthonormal basis of $B_{1}$. We have 
$\bar{e}_{k} \cdot x \in Z(\mathfrak{g})^{\perp}$, and $\left\langle\bar{e}_{k} \cdot x, b_{i}\right\rangle=-\frac{1}{2}\left\langle\left[x, b_{i}\right], \bar{e}_{k}\right\rangle$. Then $\bar{e}_{k} \cdot x=e_{0}-\frac{1}{2} \sum_{i=1}^{r}\left\langle\left[x, b_{i}\right], \bar{e}_{k}\right\rangle b_{i}$, where $e_{0} \in Z(\mathfrak{g}) \cap Z(\mathfrak{g})^{\perp}$. Using the fact that $x . b_{i}=\frac{1}{2}\left[x, b_{i}\right]$, then

$$
\begin{aligned}
x .\left(\bar{e}_{k} \cdot x\right) & =-\frac{1}{2} \sum_{i=1}^{r}\left\langle\left[x, b_{i}\right], \bar{e}_{k}\right\rangle x . b_{i} \\
& =-\frac{1}{4} \sum_{i=1}^{r}\left\langle\left[x, b_{i}\right], \bar{e}_{k}\right\rangle\left[x, b_{i}\right] .
\end{aligned}
$$

Finally, we get $\sum_{i=1}^{r}\left\langle\left[x, b_{i}\right], \bar{e}_{k}\right\rangle^{2}=0$. Since $\left[x, b_{i}\right] \in Z(\mathfrak{g}) \cap Z(\mathfrak{g})^{\perp}$, thus $Z(\mathfrak{g})^{\perp}$ is abelian.

Suppose that $\operatorname{dim} Z(\mathfrak{g}) \cap Z(\mathfrak{g})^{\perp}=1$. Then the decomposition (5.2) becomes

$$
\mathfrak{g}=Z_{1} \oplus \mathbb{R} e \oplus B_{1} \oplus \mathbb{R} \bar{e},
$$

and the restriction of $\langle$,$\rangle to Z_{1}$ and $B_{1}$ is nondegenerate.

Proposition 5.4. Let $(\mathfrak{g},\langle\rangle$,$) be a flat pseudo-Euclidean 2-step nilpotent Lie algebra such that$ $\operatorname{dim}\left(Z(\mathrm{~g}) \cap Z(\mathfrak{g})^{\perp}\right)=1$. With notations as in (5.3), if the restriction of the metric $\langle$,$\rangle to B_{1}$ is positive or negative definite, then $\operatorname{dim} B_{1}=1$, and $\mathfrak{g}$ is an extension trivial of $\mathcal{H}_{3}$, where $\mathcal{H}_{3}$ is the 3-dimensional Heisenberg Lie algebra.

Proof. Let $z \in Z(\mathfrak{g})$, and $b \in B_{1}$. We have $z . \bar{e} \in B_{1}$ and $z . b \in Z(\mathfrak{g})^{\perp}$. Since $\langle,\rangle_{B_{1} \times B_{1}}$ is positive definite or negative definite and $\langle z \cdot \bar{e}, z \cdot \bar{e}\rangle=0$, then $z \cdot \bar{e}=0$. Thus $\langle z \cdot b, \bar{e}\rangle=0$, which implies that $z . b \in B_{1}$. Using the same argument, then we can conlude that $z . b=0$, and $\mathrm{L}_{z}=0$ for any $z \in Z(\mathfrak{g})$. Let $x, y \in B_{1}$. We have for any $z \in Z(\mathfrak{g})$

$$
\langle[x, y], z\rangle=2\langle x \cdot y, z\rangle=0,
$$

thus $[x, y]=\alpha e$, where $\alpha \in \mathbb{R}$. Using the flatness of the metric, then we get $[\bar{e}, x] \cdot x=\bar{e} .(x . x)-$ $x .(\bar{e} . x)$, thus $x .(\bar{e} . x)=0$. Let $\left\{b_{1}, \ldots, b_{r}\right\}$ be an orthonormal basis of $B_{1}$. Then

$$
\bar{e} . x=\beta e \mp \frac{1}{2} \sum_{i=1}^{r}\left\langle\left[x, b_{i}\right], \bar{e}\right\rangle b_{i}
$$

where $\beta \in \mathbb{R}$. Thus

$$
x .(\bar{e} . x)=\mp \frac{1}{4} \sum_{i=1}^{r}\left\langle\left[x, b_{i}\right], \bar{e}\right\rangle\left[x, b_{i}\right]=0,
$$

which implies that $B_{1}$ is abelian. On the other hand, we have for any $z \in Z(\mathfrak{g})$,

$$
0=\langle z . \bar{e}, x\rangle=-\frac{1}{2}\langle[\bar{e}, x], z\rangle,
$$

thus $[\bar{e}, x] \in Z(\mathfrak{g}) \cap Z(\mathfrak{g})^{\perp}$. Put $\left[\bar{e}, b_{i}\right]=\alpha_{i} e$, where $\alpha_{i} \in \mathbb{R}^{*}$ for any $i \in\{1, \ldots, r\}$. In fact, if $\alpha_{i}=0$ then $b_{i} \in Z(\mathrm{~g})$, which contradicts the fact that $Z(\mathrm{~g}) \cap B_{1}=\{0\}$. Suppose that $\operatorname{dim} B_{1}>1$. For any $i \in\{2, \ldots, r\}$, we put $b_{i}^{\prime}=b_{i}-\frac{\alpha_{i}}{\alpha_{1}} b_{1}$, thus $\left[\bar{e}, b_{i}^{\prime}\right]=0$ and $b_{i}^{\prime} \in Z(\mathfrak{g})$ which is a Contradiction. Then $\operatorname{dim} B_{1}=1$ and the only non vanishing brackets in $\mathfrak{g}$ is $\left[\bar{e}, b_{1}\right]=\alpha_{1} e$, thus $\mathfrak{g}$ is an extension trivial of $\mathcal{H}_{3}$. 


\section{Flat pseudo-Euclidean 2-step nilpotent Lie algebras of signature $(2, n-2)$}

Let us start by an example which play an important role in this section. Let $\mathrm{L}_{6}^{4}$ be a 6dimenional Lie algebra defined by the non vanishing Lie brackets, giving in the basis $\left\{x_{1}, \ldots, x_{6}\right\}$ by

$$
\left[x_{1}, x_{2}\right]=x_{5},\left[x_{1}, x_{3}\right]=\left[x_{2}, x_{4}\right]=x_{6} .
$$

This Lie algebra appear in the classification of 2-step nilpotent Lie algebras of dimension 6, as for example in [1, pp.3], or in [9, pp.97], where it is denoted by $L_{6,3}$.

It is clear that this Lie algebra admits no flat Euclidean or Lorentzian metrics. However, $\mathrm{L}_{6}^{4}$ admits a flat pseudo-Euclidean metrics of signature $(2, n-2)$. In fact, let $\langle,\rangle_{0}$ be a pseudoEuclidean metric of signature $(2,4)$ defined in the basis $\left\{x_{1}, \ldots, x_{6}\right\}$ by the matrix

$$
\langle,\rangle_{0}=\left(\begin{array}{cccccc}
0 & 0 & 0 & 0 & a & 1 \\
0 & 0 & b & c & 0 & 0 \\
0 & b & 0 & 0 & 0 & 0 \\
0 & c & 0 & d & 0 & 0 \\
a & 0 & 0 & 0 & \frac{1}{3 d} & 0 \\
1 & 0 & 0 & 0 & 0 & 0
\end{array}\right)
$$

where $a, c \in \mathbb{R}, b \in \mathbb{R}^{*}$ and $d>0$. A straightforward calculations using (2.1) shows that, the only non vanishing Levi-Civita products are

$$
\begin{aligned}
& x_{1} \cdot x_{1}=-\frac{1}{b} x_{2}-\left(\frac{a}{b}+\frac{c^{2}}{b^{2} d}\right) x_{3}+\frac{c}{b d} x_{4}, x_{1} \cdot x_{2}=\frac{c}{2 b d} x_{3}-\frac{1}{2 d} x_{4}+\frac{1}{2} x_{5}+\frac{a}{2} x_{6}, \\
& x_{1} \cdot x_{3}=x_{6}, x_{1} \cdot x_{4}=\frac{1}{2 b} x_{3}, x_{1} \cdot x_{5}=x_{5} \cdot x_{1}=-\frac{1}{6 b d} x_{3}, x_{2} \cdot x_{4}=\frac{1}{2} x_{6}, x_{2} \cdot x_{5}=x_{5} \cdot x_{2}=\frac{1}{6 d} x_{6}, \\
& x_{2} \cdot x_{1}=\frac{c}{2 b d} x_{3}-\frac{1}{2 d} x_{4}-\frac{1}{2} x_{5}+\frac{a}{2} x_{6}, x_{4} \cdot x_{1}=\frac{1}{2 b} x_{3}, x_{4} \cdot x_{2}=-\frac{1}{2} x_{6} .
\end{aligned}
$$

One can verify that for any $x, y \in \mathrm{L}_{6}^{4}$, we have $\mathrm{L}_{[x, y]}=\left[\mathrm{L}_{x}, \mathrm{~L}_{y}\right]$, which shows that $\left(\mathrm{L}_{6}^{4},\langle,\rangle_{0}\right)$ is flat. The following Theorem shows that this example, is the only non trivial one such that $\operatorname{dim} Z(\mathfrak{g}) \cap Z(\mathfrak{g})^{\perp}=1$.

Let $(\mathfrak{g},\langle\rangle$,$) be a flat pseudo-Euclidean 2-step nilpotent Lie algebra of signature (2, n-2)$. According to theorem 3.1 the dimension of $Z(\mathfrak{g}) \cap Z(\mathfrak{g})^{\perp}$ is 1 or 2 .

Theorem 6.1. A 2-step nilpotent Lie algebra g admits a flat pseudo-Euclidean metric of signature $(2, n-2)$ such that $\operatorname{dim} Z(\mathfrak{g}) \cap Z(\mathfrak{g})^{\perp}=1$ if and only if $\mathfrak{g}$ is an extension trivial of $\mathcal{H}_{3}$ or $\mathfrak{g}$ is an extension trivial of $\mathrm{L}_{6}^{4}$. Furthermore, in the second case, the restriction of the metric to $\mathrm{L}_{6}^{4}$ is giving by $\langle,\rangle_{0}$.

Proof. If $\operatorname{dim} Z(\mathfrak{g}) \cap Z(\mathfrak{g})^{\perp}=1$, then we can split $\mathfrak{g}$ as

$$
\mathfrak{g}=Z_{1} \oplus \mathbb{R} e \oplus B_{1} \oplus \mathbb{R} \bar{e}
$$

where $Z(\mathfrak{g})=Z_{1} \oplus \mathbb{R} e, Z(\mathfrak{g})^{\perp}=\mathbb{R} e \oplus B_{1}, \operatorname{span}\{e, \bar{e}\}=\left(Z_{1} \oplus B_{1}\right)^{\perp},\langle e, e\rangle=\langle\bar{e}, \bar{e}\rangle=0$ and $\langle e, \bar{e}\rangle=1$. We have two cases:

First case: $\langle,\rangle /_{B_{1} \times B_{1}}$ is positive or negative definite. Then according to proposition 5.4, $\operatorname{dim} B_{1}=1$ and $g$ is an extension trivial of $\mathcal{H}_{3}$. 
Second case: $\left.\langle\rangle\right|_{,B_{1} \times B_{1}}$ is Lorentzian. Then $\operatorname{dim} B_{1} \geq 2$. For any $z, z^{\prime} \in Z(\mathfrak{g})$, we have $\left\langle z . \bar{e}, z^{\prime} \cdot \bar{e}\right\rangle=0$, then $\mathrm{R}_{\bar{e}}(Z(\mathfrak{g}))$ is a totally isotropic subspace. Since $\mathrm{R}_{\bar{e}}(Z(\mathfrak{g})) \subset B_{1}$ and $\left(B_{1},\langle,\rangle / B_{1} \times B_{1}\right)$ is Lorentzian, then there exists an isotropic vector $b_{0} \in B_{1}$ and a covector $\lambda \in Z(\mathfrak{g})^{*}$ such that $z . \bar{e}=\lambda(z) b_{0}$ for any $z \in Z(\mathfrak{g})$.

Let $x, y \in Z(\mathfrak{g})^{\perp}$. Recall that $x . y=\frac{1}{2}[x, y]$ and $\langle[x, y],[x, y]\rangle=0$. Since $Z_{1}$ is Euclidean then $[x, y] \in Z(\mathfrak{g}) \cap Z(\mathfrak{g})^{\perp}$. Choose a basis $\left\{b_{0}, \bar{b}, b_{1}, \ldots, b_{r}\right\}$ of $B_{1}$ such that $\left\{b_{1}, \ldots, b_{r}\right\}$ is orthonormal, $\operatorname{span}\left\{b_{0}, \bar{b}\right\}$ and $\operatorname{span}\left\{b_{1}, \ldots, b_{r}\right\}$ are orthogonal, $\bar{b}$ is isotropic and $\left\langle b_{0}, \bar{b}\right\rangle=1$. Then for any $i \in\{0,1, \ldots, r\}$, we have from 2.1)

$$
\left\langle\left[\bar{e}, b_{i}\right] . \bar{e}, b_{i}\right\rangle=-\frac{1}{2}\left\langle\left[\bar{e}, b_{i}\right],\left[\bar{e}, b_{i}\right]\right\rangle .
$$

On the other hand, we have $\left\langle\left[\bar{e}, b_{i}\right] . \bar{e}, b i\right\rangle=\left\langle\lambda\left(\left[\bar{e}, b_{i}\right]\right) b_{0}, b_{i}\right\rangle=0$, then $\left[\bar{e}, b_{i}\right] \in Z(\mathfrak{g}) \cap Z(\mathfrak{g})^{\perp}$.

We can write from the condition of flatness, for any $x, y, z \in \mathfrak{g}$

$$
[x, y] . z=x \cdot(y \cdot z)-y \cdot(x . z) .
$$

If we take $x=\bar{e}$ and $y=z=b_{0}$, we get $b_{0} \cdot\left(\bar{e} . b_{0}\right)=0$. Let $i \in\{0,1, \ldots, r\}$, since $\bar{e} \cdot b_{0} \in Z(\mathfrak{g})^{\perp}$ and $\left\langle\bar{e} . b_{0}, b_{i}\right\rangle=-\frac{1}{2}\left\langle\left[b_{0}, b_{i}\right], \bar{e}\right\rangle$, thus

$$
\bar{e} . b_{0}=\alpha e+\beta b_{0}-\frac{1}{2} \sum_{i=1}^{r}\left\langle\left[b_{0}, b_{i}\right], \bar{e}\right\rangle,
$$

where $\alpha, \beta \in \mathbb{R}$. It follows that $b_{0} \cdot\left(\bar{e} . b_{0}\right)=-\frac{1}{4} \sum_{i=1}^{r}\left\langle\left[b_{0}, b_{i}\right], \bar{e}\right\rangle\left[b_{0}, b_{i}\right]$, thus $\sum_{i=1}^{r}\left\langle\left[b_{0}, b_{i}\right], \bar{e}\right\rangle^{2}=0$ which implies that $\left[b_{0}, b_{i}\right]=0$ for any $i \in\{0,1, \ldots, r\}$.

If we take in (6.1), $x=\bar{e}, y=b_{0}$ and $z=\bar{b}$ we get $b_{0} \cdot(\bar{e} \cdot \bar{b})=0$. Using the fact that $b_{0} \cdot u=0$ for any $u \in Z(\mathfrak{g})$, we deduce that $b_{0} \cdot(\bar{e} \cdot \bar{b})=-\frac{1}{4}\left\langle\left[\bar{b}, b_{0}\right], \bar{e}\right\rangle\left[\bar{b}, b_{0}\right]$, thus $\left[\bar{b}, b_{0}\right]=0$. Similarly, for any $i \in\{1, \ldots, r\}$, if we take in 6.1), $x=\bar{e}$ and $y=z=e_{i}$ we get

$$
0=b_{i} \cdot\left(\bar{e} \cdot b_{i}\right)=-\frac{1}{4} \sum_{j=1}^{r}\left\langle\left[b_{i}, b_{j}\right], \bar{e}\right\rangle\left[b_{i}, b_{j}\right],
$$

thus $\left[b_{i}, b_{j}\right]=0$ for any $i, j \in\{1, \ldots, r\}$. It follows that $\operatorname{span}\left\{b_{0}, b_{1}, \ldots, b_{r}\right\}$ is abelian and $\left[b_{0}, \bar{b}\right]=$ 0 . We put

$$
\left[\bar{e}, b_{i}\right]=\alpha_{i} e,[\bar{e}, \bar{b}]=\alpha e+z_{0},\left[\bar{b}, b_{i}\right]=\beta_{i} e,
$$

where $\alpha_{i}, \beta_{i}, \alpha \in \mathbb{R}, z_{0} \in Z_{1}$ and $i=0,1, \ldots, r$. If we take in 6.1,$x=\bar{e}$ and $y=z=\bar{b}$ we get $z_{0} . \bar{b}=-\bar{b}(\bar{e} \cdot \bar{b})$, then $\frac{3}{2} z_{0} \cdot \bar{b}-\frac{1}{2} \sum_{i=1}^{r} \beta_{i}^{2} e=0$, which implies that

$$
3\left\langle z_{0}, z_{0}\right\rangle=\sum_{i=1}^{r} \beta_{i}^{2}
$$

We have $\operatorname{dim} B_{1} \geq 3$. In fact, if $\operatorname{dim} B_{1}=2$ then $B_{1}=\operatorname{span}\left\{b_{0}, \bar{b}\right\}$ and $(6.2)$ implies that $z_{0}=0$. Then the Lie brackets are reduced to $\left[\bar{e}, b_{i}\right]=\alpha_{i} e$ and $[\bar{e}, \bar{b}]=\alpha e$, and as in the proof of proposition 5.4 we can deduce that $\operatorname{dim} B_{1}=1$, which is a contradiction. The same argument shows that $z_{0} \neq 0$. Then there exists $i \in\{1, \ldots, r\}$ such that $\beta_{i} \neq 0$. To simplify, we can suppose that $\beta_{1} \neq 0$, and we have also $\alpha_{0} \neq 0$ because $b_{0} \notin Z(\mathfrak{g})$.

Let us show that $\operatorname{dim} B_{1}=3$. In fact, if $\operatorname{dim} B_{1} \geq 4$, then we put for any $i \geq 4$,

$$
b_{i}^{\prime}=b_{i}-\frac{\beta_{i}}{\beta_{1}} b_{1}-\left(\frac{\alpha_{i} \beta_{1}-\alpha_{1} \beta_{i}}{\alpha_{0} \beta_{1}}\right) b_{0},
$$


and we can verify easly that $\left[b_{i}^{\prime}, x\right]=0$ for any $x \in \mathfrak{g}$. Thus $b_{i}^{\prime} \in Z(\mathfrak{g})$ which contradicts the fact that $Z(\mathrm{~g}) \cap B_{1}=\{0\}$. We put $x_{1}=\bar{e}, x_{2}=\bar{b}, x_{3}=\frac{b_{0}}{\alpha_{0}}, x_{4}=\frac{1}{\beta_{1}} b_{1}-\frac{\alpha_{1}}{\beta_{1} \alpha_{0}} b_{0}, x_{5}=\alpha e+z_{0}$ and $x_{6}=e$. Then the only non vanishing brackets on $\mathfrak{g}$ are

$$
\left[x_{1}, x_{2}\right]=x_{5},\left[x_{1}, x_{3}\right]=\left[x_{2}, x_{4}\right]=x_{6} .
$$

It follows that $\mathrm{g}$ is an extension trivial of $\mathrm{L}_{6}^{4}$. Furthermore, with the condition (6.2), one can verify that the restriction of the metric to $\mathrm{L}_{6}^{4}$ is given by $\langle,\rangle_{0}$. Conversely, if $\mathrm{g}$ splits orthogonaly into $\mathrm{g}=Z_{1} \oplus \mathrm{L}_{6}^{4}$, where $Z_{1} \subset Z(\mathrm{~g})$ and the restriction of the metric to $\mathrm{L}_{6}^{4}$ is $\langle,\rangle_{0}$, and the restriction to $Z_{1}$ is Euclidean, then $(\mathfrak{g},\langle\rangle$,$) is a flat pseudo-Euclidean 2-step nilpotent Lie algebra of signature$ $(2, n-2)$ and $\operatorname{dim} Z(\mathfrak{g}) \cap Z(\mathfrak{g})^{\perp}=1$.

Corollary 6.1. The Heisenberg Lie algebra $\mathcal{H}_{2 k+1}$ admits a flat pseudo-Euclidean metric of signature $(2, n-2)$ if and only if $k=1$.

Proof. Since $Z\left(\mathcal{H}_{2 k+1}\right)=1$, then if $\mathfrak{g}=\mathcal{H}_{2 k+1}$ admits such metric then we have $\operatorname{dim} Z(\mathfrak{g}) \cap Z(\mathfrak{g})^{\perp}=$ 1. This gives the result, by virtue of Theorem 6.1

Remark 3. In theorem 6.1] if $\mathfrak{g}$ is an extension trivial of $\mathcal{H}_{3}$, then $\mathfrak{g}=Z_{1} \oplus \mathcal{H}_{3}$ and the metric $\langle$,$\rangle has one of the following form:$

- The restriction of $\langle$,$\rangle to Z_{1}$ is Euclidean and its restriction to $\mathcal{H}_{3}$ is given by the matix

$$
\left(\begin{array}{ccc}
0 & 0 & \alpha \\
0 & -1 & 0 \\
\alpha & 0 & 0
\end{array}\right) \text {, where } \alpha \in \mathbb{R} \text {. }
$$

- The restriction of $\langle$,$\rangle to Z_{1}$ is Lorentzian and its restriction to $\mathcal{H}_{3}$ is given by the matix

$$
\left(\begin{array}{ccc}
0 & 0 & \alpha \\
0 & 1 & 0 \\
\alpha & 0 & 0
\end{array}\right) \text {, where } \alpha \in \mathbb{R} .
$$

Theorem 6.2. A 2-step nilpotent Lie algebra $\mathfrak{g}$ admits a flat pseudo-Euclidean metric $\langle$,$\rangle of$ signature $(2, n-2)$ such that $\operatorname{dim} Z(\mathfrak{g}) \cap Z(\mathfrak{g})^{\perp}=2$ if and only if there exist an orthonormal vectors $\left\{b_{1}, \ldots, b_{k}\right\}$ in $\mathfrak{g}$, a linearly independent isotropic vectors $\left\{e_{1}, \bar{e}_{1}, e_{2}, \bar{e}_{2}\right\}$ in $\left\{b_{1}, \ldots, b_{k}\right\}^{\perp}$, where $\left\langle e_{1}, e_{2}\right\rangle=\left\langle e_{1}, \bar{e}_{2}\right\rangle=\left\langle\bar{e}_{1}, e_{2}\right\rangle=\left\langle\bar{e}_{1}, \bar{e}_{2}\right\rangle=0$ and $\left\langle e_{1}, \bar{e}_{1}\right\rangle=\left\langle e_{2}, \bar{e}_{2}\right\rangle=1$, such that for any $i \in\{1, \ldots, k\}$ the only non vanishing brackets are

$$
\begin{aligned}
& {\left[\bar{e}_{1}, \bar{e}_{2}\right]=z_{0},} \\
& {\left[\bar{e}_{1}, b_{i}\right]=\alpha_{i} e_{1}+\beta_{i} e_{2},} \\
& {\left[\bar{e}_{2}, b_{i}\right]=\gamma_{i} e_{1}+\delta_{i} e_{2},}
\end{aligned}
$$

where $\alpha_{i}, \beta_{i}, \gamma_{i}, \delta_{i} \in \mathbb{R}$, and

$$
3\left\langle z_{0}, z_{0}\right\rangle=\sum_{i=1}^{k}\left(\gamma_{i}+\beta_{i}\right)^{2}-4 \alpha_{i} \delta_{i}
$$


Proof. According to proposition 5.3, $Z(\mathfrak{g})^{\perp}$ is abelian, and we can split $\mathfrak{g}$ into

$$
\mathfrak{g}=Z_{1} \oplus \operatorname{span}\left\{e_{1}, e_{2}\right\} \oplus B_{1} \oplus \operatorname{span}\left\{\bar{e}_{1}, \bar{e}_{2}\right\}
$$

where $Z(\mathfrak{g})=Z_{1} \oplus \operatorname{span}\left\{e_{1}, e_{2}\right\}, Z(\mathfrak{g})^{\perp}=\operatorname{span}\left\{e_{1}, e_{2}\right\} \oplus B_{1},\left(Z_{1} \oplus B_{1}\right)^{\perp}=\operatorname{span}\left\{e_{1}, e_{2}, \bar{e}_{1}, \bar{e}_{2}\right\}$, $\operatorname{span}\left\{\bar{e}_{1}, \bar{e}_{2}\right\}$ is totally isotropic, $\left\langle e_{1}, e_{2}\right\rangle=\left\langle e_{1}, \bar{e}_{2}\right\rangle=\left\langle\bar{e}_{1}, e_{2}\right\rangle=\left\langle\bar{e}_{1}, \bar{e}_{2}\right\rangle=0$ and $\left\langle e_{1}, \bar{e}_{1}\right\rangle=$ $\left\langle e_{2}, \bar{e}_{2}\right\rangle=1$.

In the proof of proposition 5.3, we have shown that for any $x, y \in Z(\mathfrak{g})^{\perp}$ and $k \in\{1,2\},[x, y]$ and $\left[\bar{e}_{k}, x\right]$ are in $Z(\mathfrak{g}) \cap Z(\mathfrak{g})^{\perp}$. Let $\left\{b_{1}, \ldots, b_{r}\right\}$ be an orthonormal basis of $B_{1}$. Then, the non vanishing brackets are:

$$
\begin{aligned}
{\left[\bar{e}_{1}, \bar{e}_{2}\right] } & =z_{0}, \\
{\left[\bar{e}_{1}, b_{i}\right] } & =\alpha_{i} e_{1}+\beta_{i} e_{2}, \\
{\left[\bar{e}_{2}, b_{i}\right] } & =\gamma_{i} e_{1}+\delta_{i} e_{2},
\end{aligned}
$$

where $z_{0} \in Z(\mathfrak{g}), \alpha_{i}, \beta_{i}, \gamma_{i}, \delta_{i} \in \mathbb{R}$ and $i=1, \ldots, r$. From (2.1) and the Lie brackets above, we have for any $u \in Z(\mathfrak{g})$ and $v \in Z(\mathfrak{g})^{\perp}, u . v=0$. Recall that $(\mathfrak{g},\langle\rangle$,$) is flat if and only if for any$ $x, y, z \in \mathfrak{g}$

$$
\mathrm{L}_{[x, y]}(z)=\left[\mathrm{L}_{x}, \mathrm{~L}_{y}\right](z) .
$$

Let $x \in Z(\mathfrak{g})+Z(\mathfrak{g})^{\perp}, y, z \in \mathfrak{g}$ and $i \in\{1,2\}$. We have $\left\langle y . z, e_{i}\right\rangle=0$, then $y . z \in Z(\mathfrak{g})+Z(\mathfrak{g})^{\perp}$. Thus $x \cdot(y \cdot z)=(y \cdot z) \cdot x=0$. On the other hand, we have $x \cdot y, y \cdot x \in Z(\mathfrak{g}) \cap Z(\mathfrak{g})^{\perp}$. Thus $(x \cdot y) \cdot z=(y \cdot x) \cdot z=$ 0 . It follows that if one of the vectors $x, y$ or $z$ is in $Z(\mathfrak{g})+Z(\mathfrak{g})^{\perp}$, then 6.6 is satisfied. Thus $(\mathfrak{g},\langle\rangle$,$) is flat if and only if$

$$
\mathrm{L}_{\left[\bar{e}_{1}, \bar{e}_{2}\right]} \bar{e}_{1}-\left[\mathrm{L}_{\bar{e}_{1}}, \mathrm{~L}_{\bar{e}_{2}}\right] \bar{e}_{1}=\mathrm{L}_{\left[\bar{e}_{1}, \bar{e}_{2}\right]} \bar{e}_{2}-\left[\mathrm{L}_{\bar{e}_{1}}, \mathrm{~L}_{\bar{e}_{2}}\right] \bar{e}_{2}=0 .
$$

Straithforward calculations using 2.1) give

$$
\begin{aligned}
& z_{0} \cdot \bar{e}_{1}=-\frac{1}{2}\left\langle z_{0}, z_{0}\right\rangle e_{2}, \bar{e}_{2} \cdot \bar{e}_{1}=-\frac{1}{2} z_{0}-\frac{1}{2} \sum_{i=1}^{r}\left(\beta_{i}+\gamma_{i}\right) b_{i}, \bar{e}_{1} \cdot \bar{e}_{1}=-\sum_{i=1}^{r} \alpha_{i} b_{i}, \\
& \bar{e}_{1} b_{i}=\alpha_{i} e_{1}+\frac{1}{2}\left(\beta_{i}+\gamma_{i}\right) e_{2}, \bar{e}_{2} b_{i}=\frac{1}{2}\left(\beta_{i}+\gamma_{i}\right) e_{1}+\delta_{i} e_{2} .
\end{aligned}
$$

Thus the condition $\mathrm{L}_{\left[\bar{e}_{1}, \bar{e}_{2}\right]} \bar{e}_{1}-\left[\mathrm{L}_{\bar{e}_{1}}, \mathrm{~L}_{\bar{e}_{2}}\right] \bar{e}_{1}=0$ is equivalent to 6.3). Similarly, we show that the second condition $\mathrm{L}_{\left[\bar{e}_{1}, \bar{e}_{2}\right]} \bar{e}_{2}-\left[\mathrm{L}_{\bar{e}_{1}}, \mathrm{~L}_{\bar{e}_{2}}\right] \bar{e}_{2}=0$ is also equivalent to (6.3). This completes the proof.

Corollary 6.2. If a 2-step nilpotent Lie algebra $\mathfrak{g}$ admits a flat pseudo-Euclidean metric of signature $(2, n-2)$, then $\operatorname{dim}[\mathfrak{g}, \mathfrak{g}] \leqslant 3$.

\section{Examples}

In this section, we show that any 6-dimensional 2-step nilpotent Lie algebra, which is not an extension trivial of $\mathcal{H}_{5}$, admits a flat pseudo-Euclidean metric of signature $(2, n-2)$, where $\mathcal{H}_{5}$ is a 5-dimensional Heisenberg Lie algebra. For this, we use the table below which give all 6-dimensional 2-step nilpotent Lie algebras (see [1, pp.3]). Note that $\mathcal{H}_{5}$ (resp. $\mathcal{H}_{3}$ ) is denoted in this table by $\mathrm{L}_{5}^{4}$ (resp. $\mathrm{L}_{3}$ ). 


\begin{tabular}{ll}
\hline Lie algebra & Nonzero commutators \\
\hline$L_{3} \oplus 3 L_{1}$ & {$\left[x_{1}, x_{2}\right]=x_{3}$} \\
$L_{5}^{1} \oplus L_{1}$ & {$\left[x_{1}, x_{2}\right]=x_{3},\left[x_{1}, x_{4}\right]=x_{5}$} \\
$L_{5}^{4} \oplus L_{1}$ & {$\left[x_{1}, x_{3}\right]=x_{5},\left[x_{2}, x_{4}\right]=x_{5}$} \\
$L_{3} \oplus L_{3}$ & {$\left[x_{1}, x_{2}\right]=x_{3},\left[x_{4}, x_{5}\right]=x_{6}$} \\
$L_{6}^{4}$ & {$\left[x_{1}, x_{2}\right]=x_{5},\left[x_{1}, x_{3}\right]=x_{6},\left[x_{2}, x_{4}\right]=x_{6}$} \\
$L_{6}^{5}(-1)$ & {$\left[x_{1}, x_{3}\right]=x_{5},\left[x_{1}, x_{4}\right]=x_{6},\left[x_{2}, x_{4}\right]=x_{5},\left[x_{2}, x_{3}\right]=-x_{6}$} \\
$L_{6}^{3}$ & {$\left[x_{1}, x_{3}\right]=x_{6},\left[x_{1}, x_{2}\right]=x_{4},\left[x_{2}, x_{3}\right]=x_{5}$} \\
\hline
\end{tabular}

The result is evident for $\mathrm{L}_{3} \oplus 3 \mathrm{~L}_{1}$ and $\mathrm{L}_{6}^{4}$ (theorem 6.1). Let $\langle$,$\rangle be a pseudo-Euclidean metric$ of signature $(2, n-2)$ given by the matrix

$$
\langle,\rangle=\left(\begin{array}{llllll}
0 & 1 & 0 & 0 & 0 & 0 \\
1 & 0 & 0 & 0 & 0 & 0 \\
0 & 0 & 0 & 1 & 0 & 0 \\
0 & 0 & 1 & 0 & 0 & 0 \\
0 & 0 & 0 & 0 & 1 & 0 \\
0 & 0 & 0 & 0 & 0 & 1
\end{array}\right)
$$

Using theorem 6.2 let us show that, for all those Lie algebras $\mathrm{L}_{5}^{1} \oplus \mathrm{L}_{1}, \mathrm{~L}_{3} \oplus \mathrm{L}_{3}, \mathrm{~L}_{6}^{5}(-1)$ and $\mathrm{L}_{6}^{3}$ there exists a basis $\mathbb{B}$ such that the metric given in $\mathbb{B}$ by $\langle$,$\rangle is flat.$

- For $\mathrm{L}_{5}^{1} \oplus \mathrm{L}_{1}$, with our notations we put $\mathbb{B}=\left\{e_{1}, \bar{e}_{1}, e_{2}, \bar{e}_{2}, z_{0}, b_{1}\right\}$ where $e_{1}=x_{6}, \bar{e}_{1}=x_{1}$, $e_{2}=x_{5}, \bar{e}_{2}=x_{2}, z_{0}=x_{3}$ and $b_{1}=x_{4}$. One can verify easly that in this basis, the Lie brackets and the metric verify the conditions (6.3) and (6.4), thus $\left(\mathrm{L}_{5}^{1} \oplus \mathrm{L}_{1},\langle\rangle,\right)$ is flat.

- For $\mathrm{L}_{3} \oplus \mathrm{L}_{3}$, we put $\mathbb{B}=\left\{e_{1}, \bar{e}_{1}, e_{2}, \bar{e}_{2}, b_{1}, b_{2}\right\}$ where $e_{1}=x_{3}, \bar{e}_{1}=x_{1}, e_{2}=x_{6}, \bar{e}_{2}=x_{4}$, $b_{1}=x_{1}$ and $b_{2}=x_{5}$.

- For $\mathrm{L}_{6}^{5}(-1)$, we put $\mathbb{B}=\left\{e_{1}, \bar{e}_{1}, e_{2}, \bar{e}_{2}, b_{1}, b_{2}\right\}$ where $e_{1}=x_{5}+x_{6}, \bar{e}_{1}=x_{1}, e_{2}=-2 x_{6}$, $\bar{e}_{2}=x_{2}, b_{1}=x_{4}$ and $b_{2}=-(3+\sqrt{15}) x_{4}-x_{3}$.

- For $\mathrm{L}_{6}^{3}$, we put $\mathbb{B}=\left\{e_{1}, \bar{e}_{1}, e_{2}, \bar{e}_{2}, z_{0}, b_{1}\right\}$ where $e_{1}=x_{4}, \bar{e}_{1}=x_{1}, e_{2}=\frac{4}{3} x_{5}, \bar{e}_{2}=x_{3}, z_{0}=x_{6}$ and $b_{1}=x_{2}$.

For $\mathfrak{g}=\mathrm{L}_{5}^{4} \oplus \mathrm{L}_{1}$, it is clear that this algebra can not admit flat pseudo-Euclidean metric of signature $(2, n-2)$ such that $\operatorname{dim} Z(\mathrm{~g}) \cap Z(\mathrm{~g})^{\perp}=1$ (theorem 6.1). Suppose that it admits such metric with $\operatorname{dim} Z(\mathfrak{g}) \cap Z(\mathfrak{g})^{\perp}=2$ (theorem 6.2$)$. We have $\operatorname{dim}[\mathfrak{g}, \mathfrak{g}]=1$ and $\operatorname{dim} Z(\mathfrak{g})=2$. Then $\operatorname{dim} Z(\mathfrak{g})^{\perp}=4$ and $\operatorname{dim} B_{1}=2$. Put $[\mathfrak{g}, \mathfrak{g}]=\mathbb{R} e_{1}$, thus the Lie brackets satisfy

$$
\left[\bar{e}_{1}, \bar{e}_{2}\right]=\alpha e_{1},\left[\bar{e}_{1}, b_{i}\right]=\alpha_{i} e_{1},\left[\bar{e}_{2}, b_{i}\right]=\gamma_{i} e_{1}, i=1,2 .
$$

The condition (6.4) implies that $\gamma_{1}=\gamma_{2}=0$. Then $\alpha, \alpha_{1}, \alpha_{2} \in \mathbb{R}^{*}$. The fact that $\alpha=0$, for example, implies that $\bar{e}_{2} \in Z(\mathfrak{g})$. Put $b_{2}^{\prime}=b_{2}-\frac{\alpha_{2}}{\alpha_{1}} b_{1}$, then $b_{2}^{\prime} \in Z(\mathfrak{g})$, which is a contradiction. It follows that $\mathrm{L}_{5}^{4} \oplus \mathrm{L}_{1}$ can not admit flat pseudo-Euclidean metrics of signature $(2, n-2)$.

\section{References}

[1] Abiev, A. N. (2014). On the ricci curvature of solvable metric Lie algebras with two-step nilpotent derived algebras, Siberian Advences in Mathematics, V. 24, No. 1, 1-11. 
[2] Aubert, A., Medina, A. (2003). Groupes de Lie Pseudo-riemanniens plats, Tohoku Math. J., V. 55, 487-506.

[3] Ait Ben Haddou, M., Boucetta, M., Lebzioui, H. (2012). Left-Invariant Lorentzian Flat Metrics on Lie Groups, Journal of Lie Theory, V. 22, 269-289.

[4] Boucetta, M., Lebzioui, H. (2016). Flat Nonunimodular Lorentzian Lie Algebras, Communications in Algebra, Volume 44, Issue 10, pp. 4185-4195.

[5] Boucetta, M. (2009). Ricci flat left-invariant pseudo-Riemannian metrics on 2-step nilpotent Lie groups, arXiv: $0910.2563 \mathrm{v} 1$ [math. D.G].

[6] Guédiri, M., Bin-Asfour, M. (2014). Ricci-flat left-invariant Lorentzian metrics on 2-step nilpotent Lie groups, Archivum Mathematicum, V. 50, No. 3, 171-192.

[7] Guédiri, M. (2003). On the nonexistence of closed timelike geodesics in flat lorentz 2-step nilmanifolds, Transactions of the American Mathematical Society, V. 355, 775-786.

[8] Milnor, J. (1976). Curvatures of left invariant metrics on Lie Groups, Advances in Mathematics 21, 293-329.

[9] Revoy, P. (1980). Algèbres de Lie métabéliennes, Annales de la faculté des sciences de Toulouse, 5éme série, tome 2 , No. $2,93-100$ 